\title{
Robust Vapor-Deposited Antifouling Fluoropolymer Coatings for Stainless Steel Polymerization Reactor Components
}

\author{
Nareh Movsesian ${ }^{1}$, Sabine Hirth ${ }^{2}$, Joshua Speros $^{3}$, and Malancha Gupta ${ }^{1 *}$
}

1. Mork Family Department of Chemical Engineering and Materials Science, University of Southern California, 925 Bloom Walk, Los Angeles, California 90089, United States

2. BASF SE, Department of Material Physics, Analytics \& Formulation, Carl-Bosch-Strasse 38, 67056 Ludwigshafen am Rhein, Germany

3. California Research Alliance (CARA), BASF Corporation, Berkeley, California 94720, United States

*Corresponding author email: malanchg@usc.edu

\section{Supplementary Information}

\section{Experimental Details of the iCVD Depositions}

For the deposition of the control pC8PFA homopolymer coating, the C8PFA jar was heated to $50^{\circ} \mathrm{C}$. C8PFA and TBPO were introduced into the reactor at 0.1 and $0.7 \mathrm{sccm}$, respectively, and the reactor pressure was maintained at 70 mtorr. The filament array was then resistively heated for 30 minutes to achieve a polymer thickness of approximately $300 \mathrm{~nm}$. For the deposition of the control pC6PFA homopolymer coating, the C6PFA jar was heated to $50^{\circ} \mathrm{C}$. C6PFA and $\mathrm{TBPO}$ were introduced into the reactor at 1.6 and $0.7 \mathrm{sccm}$, respectively, and the reactor pressure was maintained at 70 mtorr. The filament array was resistively heated for 16 minutes to achieve a thickness of approximately $300 \mathrm{~nm}$. For the deposition of the p(C8PFA-co-EGDA) coating, 
C8PFA and EGDA were heated to $50^{\circ} \mathrm{C}$ and $35^{\circ} \mathrm{C}$, respectively. C8PFA, EGDA, and TBPO were introduced into the reactor at flow rates of $0.05,0.4$, and $0.7 \mathrm{sccm}$, respectively, and the reactor pressure was maintained at 70 mtorr. The filament array was resistively heated for 12 minutes to achieve a thickness of approximately $300 \mathrm{~nm}$. For the deposition of the p(C6PFA-co-EGDA) coating, the C6PFA and EGDA were heated to $25^{\circ} \mathrm{Cand} 35^{\circ} \mathrm{C}$, respectively. C6PFA, EGDA, and TBPO were introduced into the reactor at flow rates of $0.2,0.4$, and $0.7 \mathrm{sccm}$, respectively, and the reactor pressure was maintained at 70 mtorr. The filament array was resistively heated for 47 minutes to achieve a thickness of approximately $300 \mathrm{~nm}$. For the deposition of the pC8PFApEGDA coating, C8PFA and EGDA were heated to $50^{\circ} \mathrm{C}$ and $40^{\circ} \mathrm{C}$, respectively. The pEGDA layer was deposited first by introducing EGDA and TBPO at 0.1 and $0.6 \mathrm{sccm}$, respectively, and the reactor pressure was maintained at 100 mtorr. The filament array was heated for 3 minutes in order to achieve a thickness of $100 \mathrm{~nm}$. Next, the filament array was turned off, the EGDA and TBPO flows were halted, and the reactor was allowed to pump down to base pressure for approximately 5 mins. The pC8PFA homopolymer was then deposited on top of the pEGDA layer by introducing C8PFA and TBPO at 0.1 and $0.7 \mathrm{sccm}$, respectively, at a reactor pressure of 70 mtorr and heating the filament array for 35 minutes in order to achieve a thickness of $300 \mathrm{~nm}$. For the deposition of the pC6PFA-pEGDA coating, C6PFA and EGDA were heated to $50^{\circ} \mathrm{C}$ and $40^{\circ} \mathrm{C}$, respectively. The pEGDA layer was deposited as described above. The pC6PFA homopolymer was then deposited on top of the pEGDA layer by introducing C6PFA and TBPO at 1.6 and 0.7 sccm, respectively, at a reactor pressure of $70 \mathrm{mtorr}$ and heating the filament array for 11 minutes in order to achieve a thickness of $300 \mathrm{~nm}$. 


\section{Supplemental Figures}

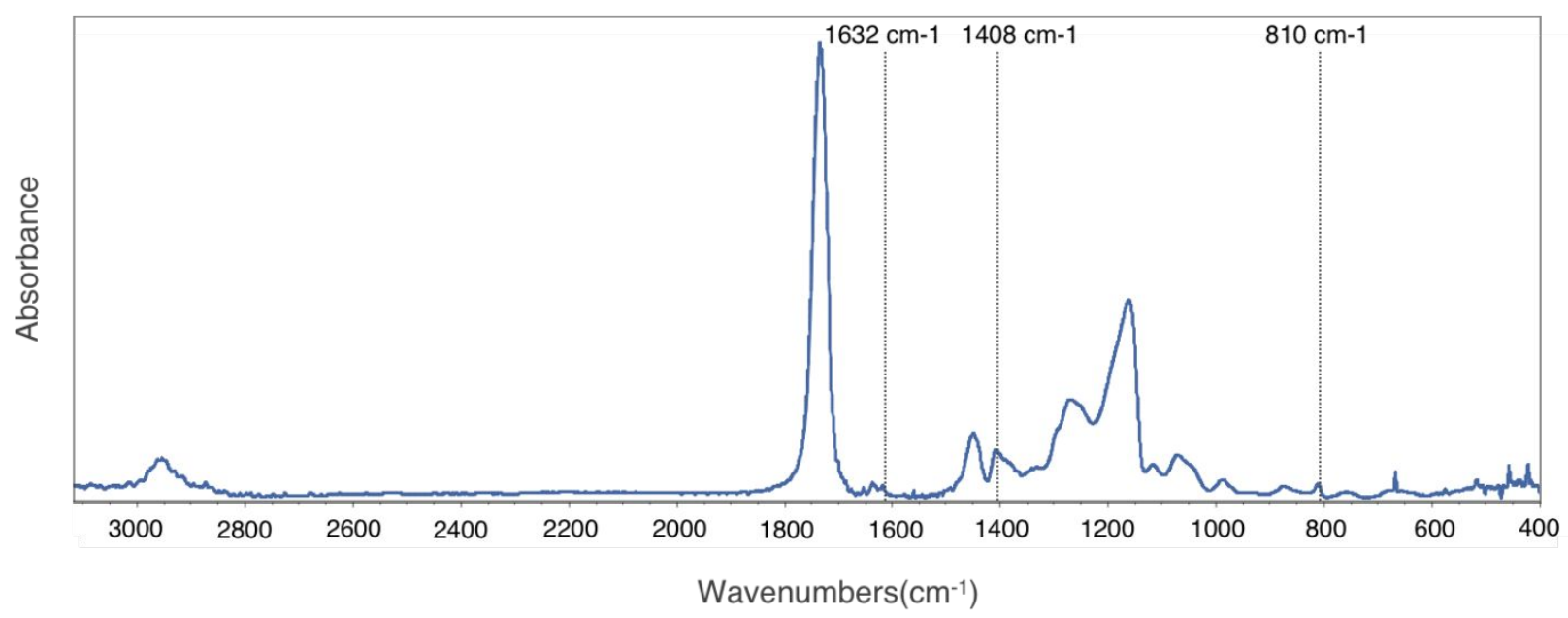

Figure S1. FTIR spectrum of the pEGDA crosslinked base layer. The assigned peaks correspond to the vibrations of the unreacted vinyl bonds. 
a)

plasma cleaned bare S.S.
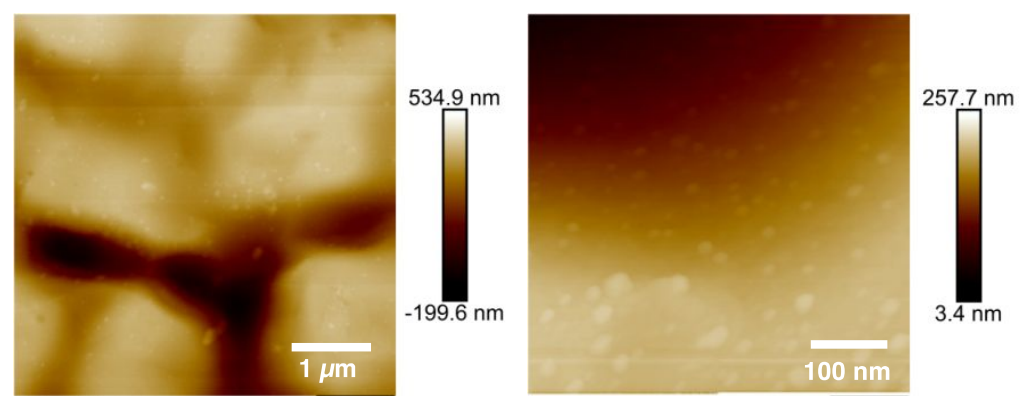

b)

bare silicon wafer

c)

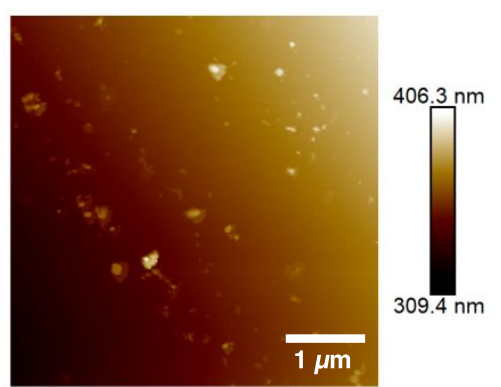

$\mathrm{p}(\mathrm{C} 8 \mathrm{PFA}-\mathrm{co}-\mathrm{EGDA})$ coated silicon wafer

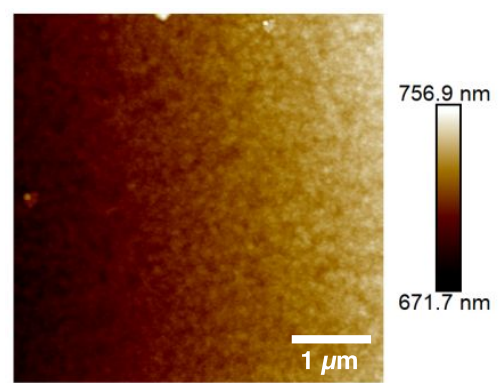

d)

$$
\text { pC8PFA-pEGDA coated silicon wafer }
$$

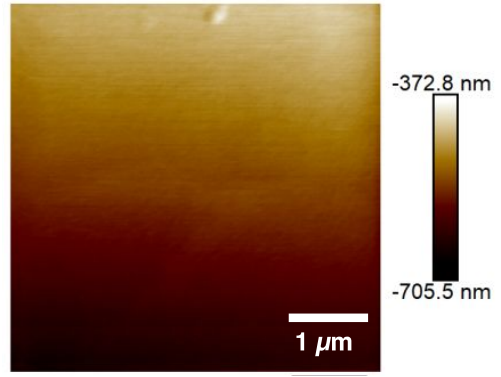

Figure S2. a) $5 \times 5 \mu \mathrm{m}^{2}$ and $500 \times 500 \mathrm{~nm}^{2}$ AFM height micrographs of plasma cleaned bare stainless steel (S.S.). $5 \times 5 \mu \mathrm{m}^{2}$ AFM height micrographs of b) bare silicon wafer, c) $\mathrm{p}(\mathrm{C} 8 \mathrm{PFA}-$ co-EGDA) coated silicon wafer, and d) pC8PFA-pEGDA coated silicon wafer. 


\section{p(C8PFA-co-EGDA) coated S.S. \\ as deposited}
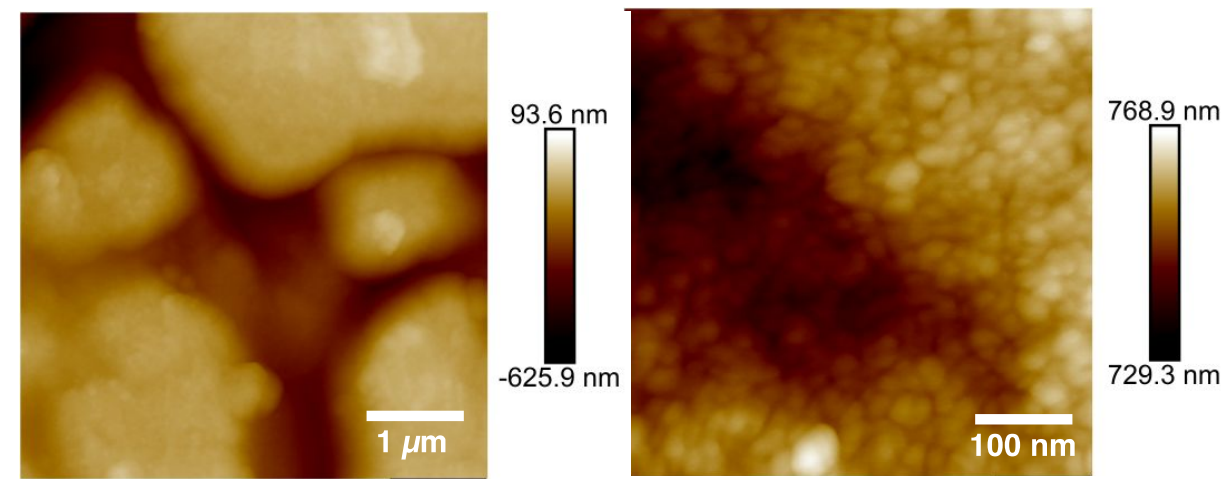

after tape test
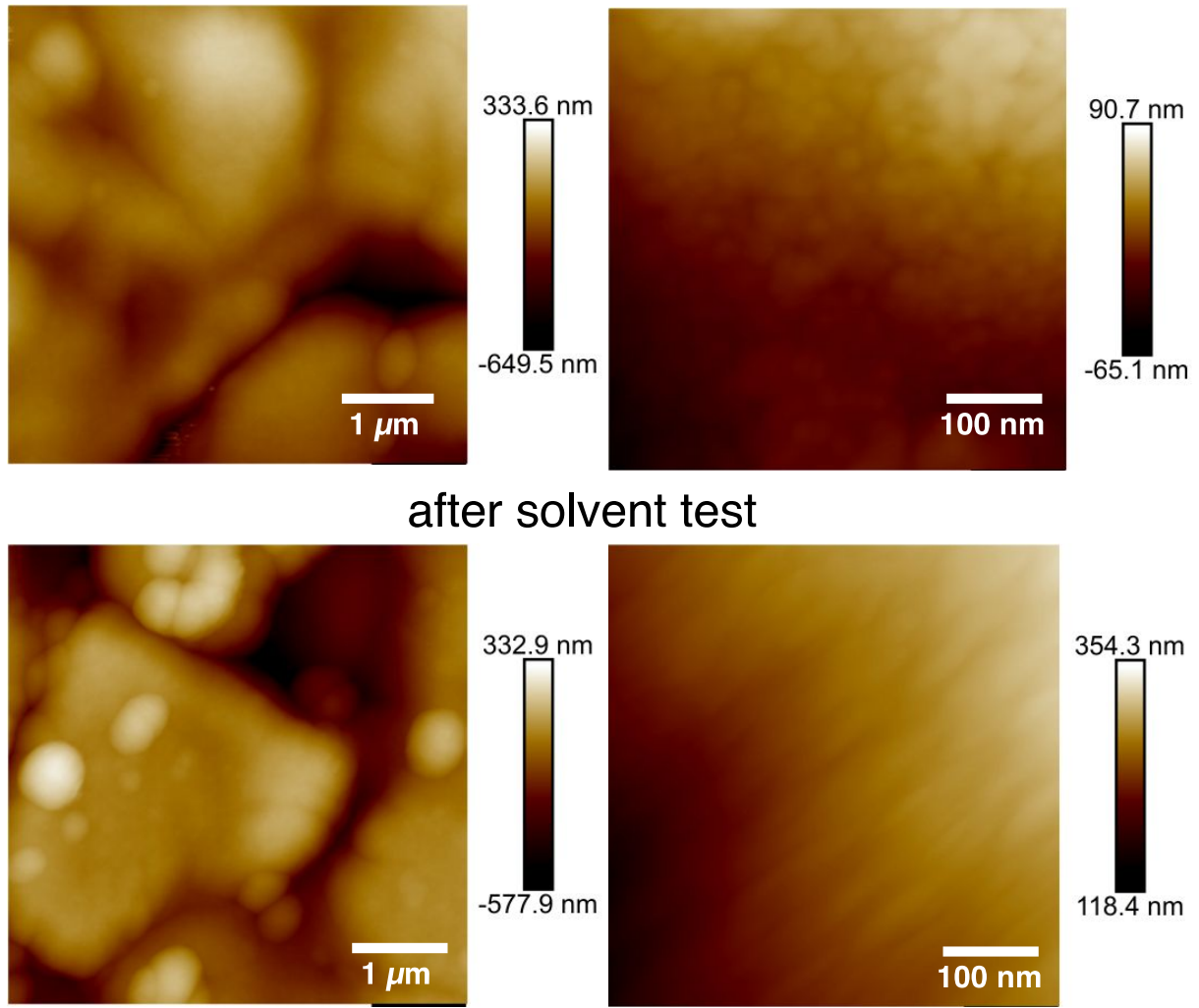

$354.3 \mathrm{~nm}$

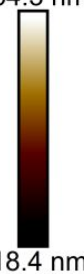

Figure S3. $5 \times 5 \mu \mathrm{m}^{2}$ and $500 \times 500 \mathrm{~nm}^{2}$ AFM height micrographs of $\mathrm{p}(\mathrm{C} 8 \mathrm{PFA}$-co-EGDA) coated S.S. as deposited, after tape test, and after solvent test. 


\section{pC8PFA-pEGDA coated S.S.}

as deposited

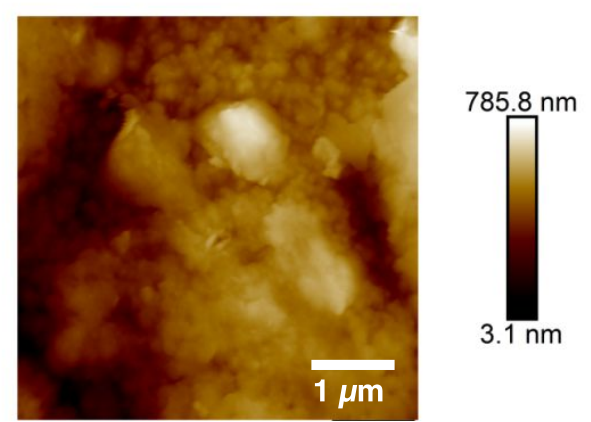

after tape test
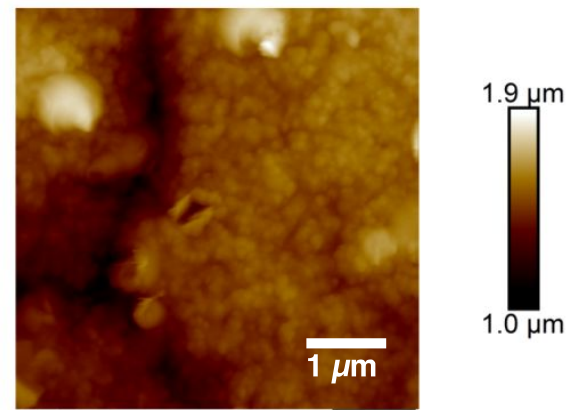

after solvent test
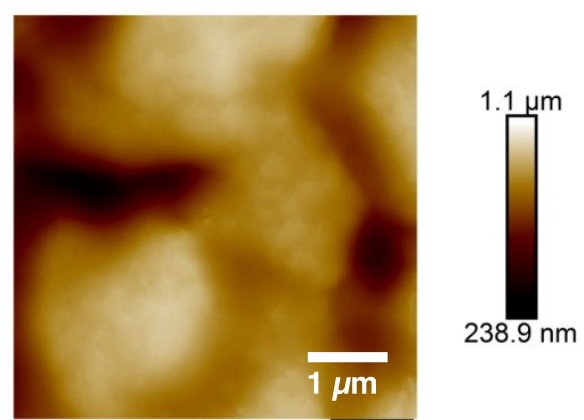

Figure S4. $5 \times 5 \mu \mathrm{m}^{2}$ AFM height micrographs of pC8PFA-pEGDA coated S.S. as deposited, after tape test, and after solvent test. 
a)

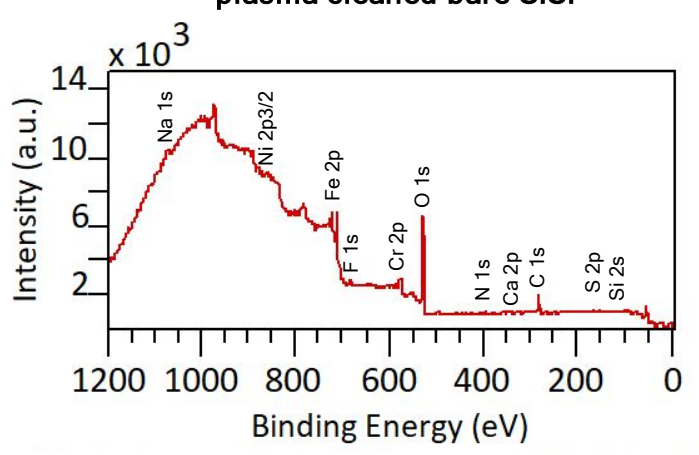

C)

p(C8PFA-co-EGDA) coated S.S. after solvent test

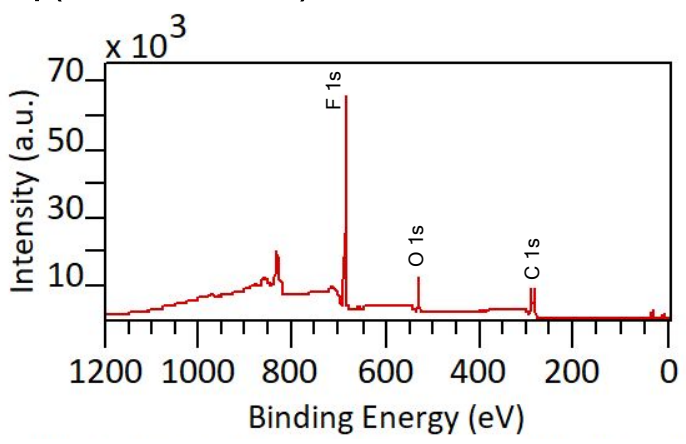

b)

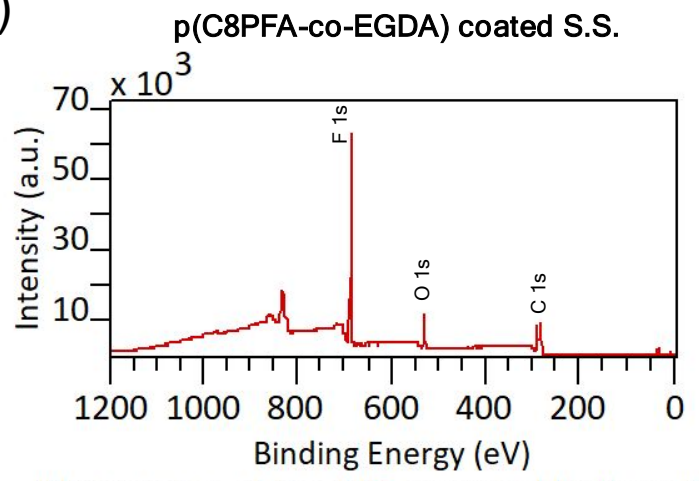

d)
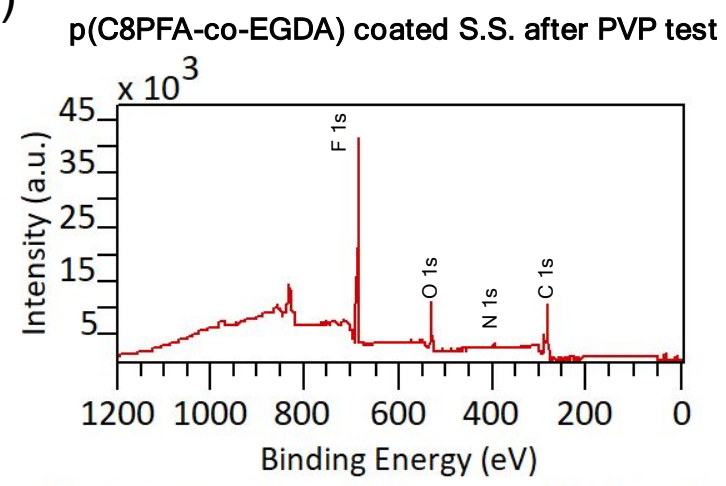

Figure S5. Representative XPS survey spectra of a) uncoated S.S., b) p(C8PFA-co-EGDA) coated S.S., c) p(C8PFA-co-EGDA) coated S.S. after solvent test, and d) p(C8PFA-co-EGDA) coated S.S. after PVP test. 
a)

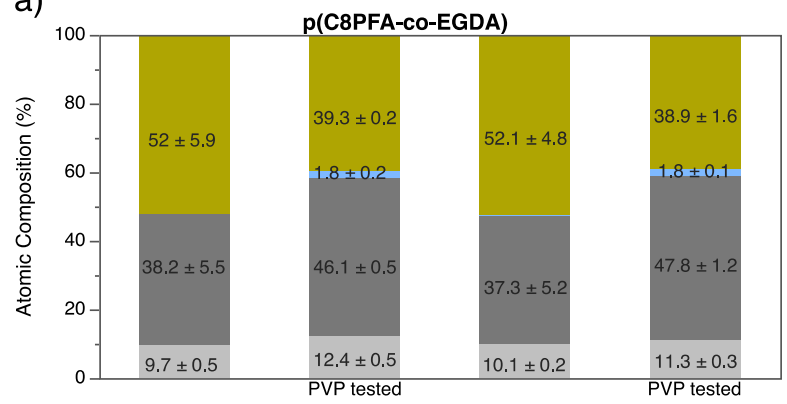

b)

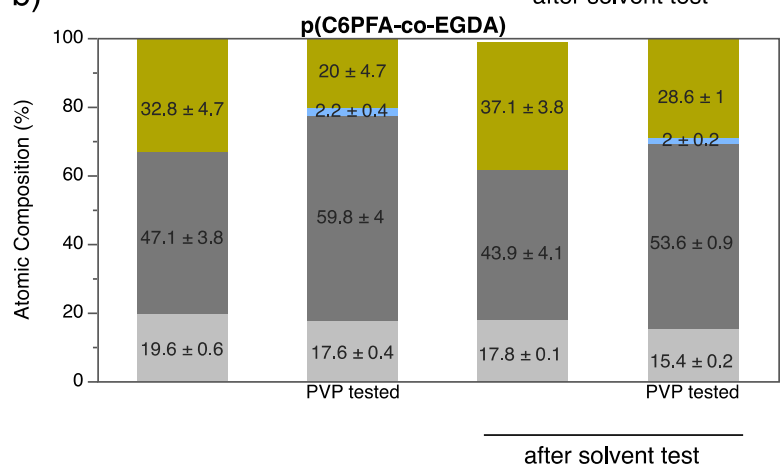

c)

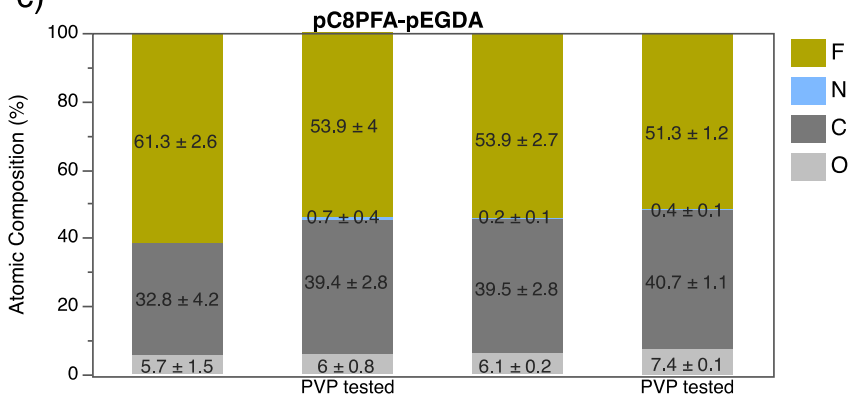

d)

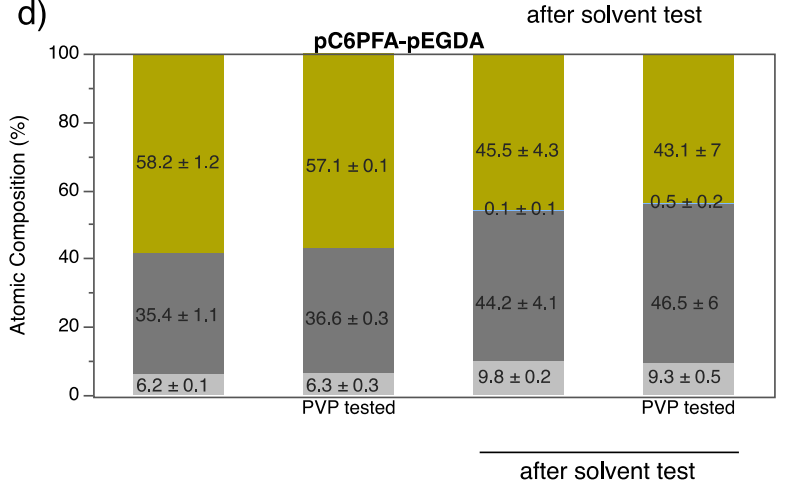

Figure S6. XPS atomic composition of a) p(C8PFA-co-EGDA), b) pC8PFA-pEGDA, c) p(C6PFA-co-EGDA), and d) pC6PFA-pEGDA before and after the PVP fouling test for the asdeposited coatings and the coatings that were solvent tested. Reported error bars represent the standard deviation within three positions in a sample. 